\title{
Cervicothoracic Epidural Blood Patch Provides Pain Relief after Failed lumbar Epidural Blood Patch: A Case Report
}

\author{
Owais Qureshi, DO, Phillip Suwan, MD, Sachin Bahadur, MD, and Anterpreet Dua, MD
}

Background: Spontaneous intracranial hypotension is an increasingly relevant cause of headaches as the breadth and depth of its diagnosis and management improves.

Objectives: The objective of this case report is to describe a novel treatment for headaches caused by spontaneous intracranial hypotension without locating the cerebrospinal fluid (CSF) leak and to review the diagnostic and therapeutic options for spontaneous intracranial hypotension.

Study Design: This is a report of a single case. Setting: The setting for this case report is a major academic center.

Methods: After a review of the treatments and interventions that our patient had previously undergone, we decided to trial epidural blood patches for her chronic headaches.

Results: Although the exact site of a CSF leak could not be identified despite exhaustive di- agnostic interventions, cervicothoracic epidural blood patches provided excellent pain control for our patient's severe headaches. The patient was completely free of headaches for 15 hours and reported well-controlled pain for approximately a month after her procedure as well as after 5 subsequent monthly procedures.

Limitations: As this is a report of a single case, more rigorous study in a larger patient population is needed to provide guidance.

Conclusions: This case demonstrates that a nontargeted cervicothoracic epidural blood patch can be a treatment option for patients with spontaneous intracranial hypotension in which clinical suspicion is high despite nondiagnostic imaging. Key words: Spontaneous intracranial hypotension, cervicothoracic epidural blood patch, headache
Spontaneous intracranial hypotension is an increasingly relevant cause of headaches as the breadth and depth of its diagnosis and management improves. Currently, the first line of therapy for spontaneous intracranial hypotension after conservative treatment is a blind epidural blood patch (EBP). If there is no significant improvement in the headache, diagnostic imaging is used to identify the exact location of the cerebrospinal fluid (CSF) leak and a targeted ap-

From : Anesthesiology and Perioperative Medicine, Medical College of Georgia, Augusta University, Georgia

Author for correspondence: Anterpreet Dua, MD

Address: Anesthesiology and Perioperative Medicine, Augusta University, 1120 15th Street BIW-2144; Augusta GA 30912

E-mail: adua@augusta.edu proach under fluoroscopic guidance is performed. If a precise location cannot be established, the therapeutic options have been limited. Herein we describe a case in which an alternative approach to the EBP was performed due to an inability to determine the exact site of the CSF leak.

\section{CASE REPORT}

A 44-year-old woman with a history of catamenial migraines was referred to our pain clinic by her primary care physician for a chronic headache of one year's duration. The patient was in her usual state until she went on her first yoga retreat. During that weekend, she developed severe headaches of a different character than her previous headaches. She described the headaches as a constant, severe, burning pain located in the occiput and extending down into the 
neck. She also stated that she felt as if her entire head were about to explode. Her pain was subdued in the mornings and gradually intensified throughout the day. Pain was also exacerbated with bending. Her pain improved in the supine position, and there was relief with caffeine intake in the morning. Her headaches were associated with photophobia, phonophobia, and nausea; but not vomiting, visual changes, weakness, or numbness.

The patient had failed numerous pharmacological therapies including topiramate, amitriptyline, imipramine, valproic acid, mirtazapine, propranolol, venlafaxine, and tizanidine. She had multiple acute treatments as well, including rizatriptan, almotriptan, and ketorolac, none of which lessened the severity of her pain. Prior interventions included bilateral occipital nerve blocks, trigger point injections into the cervical paraspinal muscles and shoulders, botulinum toxin injections, and bilateral sphenopalatine ganglion blocks with no relief. Most recently, she had an empiric lumbar EBP with partial immediate relief that subsided once she resumed physical activity.

The patient underwent magnetic resonance imaging (MRI) of the brain and of the cervical, thoracic, and lumbar spine; the MRI revealed a syrinx at approximately T8-T11 and small arachnoid cysts at the nerve root sleeves at multiple levels throughout the cervical, thoracic, and lumbar regions. A lumbar puncture was subsequently performed, which demonstrated an opening CSF pressure of 8-9 mm Hg. Radionuclide cisternography and myelography results were unremarkable. At our clinic, a C7-T1 $25 \mathrm{~mL}$ autologous blood patch and a T10-T11 $15 \mathrm{~mL}$ autologous blood patch were performed under anteroposterior and lateral fluoroscopic guidance in the prone position. The patient remained in the recumbent position for 36 hours after the procedure. The patient had marked improvement in symptoms from her initial severe pain to a grading of "mild to moderate" pain. Her numerical rating system score decreased from a 7 prior to the procedure to a zero and she remained headache free for 15 hours. The patient's pain was well-controlled for approximately one month, when she was treated with another blood patch with a similar level of relief. The patient continued receiving blood patches monthly for 5 months with either marked improvement or complete relief at every visit.

\section{DISCUSSION}

Spontaneous intracranial hypotension is a diagnostically and therapeutically challenging headache syndrome. Spontaneous intracranial hypotension most commonly causes a postural-type headache located in the occipital region similar to our case (2). It is now thought that the postural component ranges with intensity and may evolve with time (3). Our patient's headache worsened throughout the day and improved overnight. The exact inciting event of dural trauma was thought to be an unclear minor stressor.

At present, there is no single test that can diagnose spontaneous intracranial hypotension with high sensitivity; therefore, different diagnostic modalities are often pursued to establish a diagnosis. The 3 most useful tests are MRI of the brain, MRI of the spine, and CSF pressure measurements (3). Brain MRI findings, although common, are not present in all patients with spontaneous intracranial hypotension (3). Findings may include but are not limited to diffuse meningeal enhancement, subdural fluid collections such as hematomas or hygromas, "sagging" of the brain with cerebellar tonsillar herniation and descent of the brainstem mimicking Chiari 1 malformation, engorgement of cerebral venous sinuses, or pituitary enlargement (1). In fact, the most common brain MRI finding, dural enhancement, has been found to decrease in prevalence with time after symptom onset (4). This may be why the brain MRI of our patient was negative, as she did not present to us until a year after her headaches began. CSF leaks arising from nerve root sleeves, identified via an MRI of the spine, are often associated with a focal outpouching of the sleeve, such as a cyst, and are a well-known cause of spontaneous intracranial hypotension (3). Findings may also include extra-arachnoid fluid collections, collapse of the dural sac and engorgement of the epidural venous plexus, meningeal diverticula, and extradural extravasation of fluid (1). Although an MRI of the patient's spine showed cysts, there was no evidence of a rapid or slow leak. The third diagnostic modality, opening lumbar CSF pressure, was also found to be normal. Typically, a CSF pressure of less than $6 \mathrm{~cm} \mathrm{H}_{2} \mathrm{O}$ supports a diagnosis (3). Thus, it was decided to perform radionuclide cisternography and myelography, as these tests are often performed when a source of CSF leak cannot be found on the MRI or when a blind lumbar blood patch fails to 
give the patient relief (1). The most common cisternographic abnormality is the absence or paucity of activity over the cerebral convexities. Other findings may include leakage of isotope outside the normal confines of the subarachnoid space (1). Computed tomography (CT) myelography is the best test to identify the exact site of a CSF leak. Both of these exams were negative and an exact site of a CSF leak was never identified. Therefore, a targeted EBP or percutaneous fibrin sealant was not therapeutically feasible in our patient (5).

Despite negative imaging studies and a failed lumbar EBP, C7-T1 and T10-T11 autologous blood patches were performed under fluoroscopic guidance and the patient found complete relief of her headache symptoms. In our case, EBP intervention was both diagnostic and therapeutic.

Advanced imaging, such as radionuclide cisternography and CT myelography are expensive, time consuming, and at times, unrevealing (3). Fluoroscopically-guided cervicothoracic EBP offers an efficient way to both diagnose and treat spontaneous intracranial hypotension and reduce costs as well. So far, reported studies have been of nontargeted lumbar EBP $(6,7,8)$. A case series with 25 patients found lumbar EBP to be successful in reducing pain in $36 \%-57 \%$ of patients on the first attempt and $33 \%-67 \%$ on a second attempt (6). Often cervical or thoracic EBP are done only if the CSF site is known (9). Although more extensive studies are certainly needed, this case demonstrates that a nontargeted cervicothoracic EBP can be a treatment option for patients with spontaneous intracranial hypotension in which clinical suspicion is high despite nondiagnostic imaging.

\section{Acknowledgments}

The authors have nothing to disclose.

\section{REFERENCES}

1. Sun-Edelstein C, Lay CL. Spontaneous intracranial hypotension: Treatment and prognosis. UpToDate.com. www.uptodate. com. Updated February 16, 2017. Accessed July 31, 2018.

2. Shear T, Ahmed SU. Epidural blood patch for chronic daily headache with postural component: A case report and the review of published cases. Pain Physician 2008; 11:77-80.

3. Kranz PG, Malinzak MD, Amrhein TJ, Gray L. Update on the diagnosis and treatment of spontaneous intracranial hypotension. Curr Pain Headache Rep 2017; 21:37.

4. Kranz PG, Amrhein TJ, Choudhury KR, Tanpitukpongse TP, Gray L. Time-dependent changes in dural enhancement associated with spontaneous intracranial hypotension. AJR Am J Roentgenol 2016; 207:1283-1287.

5. Schievink WI. Spontaneous spinal cerebrospinal fluid leaks and intracranial hypotension. JAMA 2006; 295:2286-2296.

6. Sencakova D, Mokri B, McClelland RL. The efficacy of epidural blood patch in spontaneous CSF leaks. Neurology 2001; 57:1921-1923.

7. Berroir S, Loisel B, Ducros A, Boukobza M, Tzourio C, Valade $D$, Bousser MG. Early epidural blood patch in spontaneous intracranial hypotension. Neurology 2004; 63:1950-1951.

8. Chung SJ, Lee JH, Im JH, Lee MC. Short-and long-term outcomes of spontaneous CSF hypovolemia. Eur Neurol 2005; 54:63-67.

9. So Y, Park JM, Lee P, Kim CL, MD, Lee C, Kim JH. Epidural blood patch for the treatment of spontaneous and iatrogenic orthostatic headache. Pain Physician 2016; 19:E1115-E1122. 
